\title{
Stable circulation modes in a dual-core matter-wave soliton laser
}

\author{
Peter Y. P. Chen \\ School of Mechanical and Manufacturing Engineering, \\ University of New South Wales, Sydney 2052, Australia \\ Boris A. Malomed \\ Department of Interdisciplinary Studies, School of Electrical Engineering, \\ Faculty of Engineering, Tel Aviv University, Tel Aviv 69978, Israel
}

\begin{abstract}
We consider a model of a matter-wave laser generating a periodic array of solitary-wave pulses. The system, a general version of which was recently proposed in Ref. [5], is composed of two parallel tunnel-coupled cigar-shaped traps (a reservoir and a lasing cavity), solitons being released through a valve at one edge of the cavity. We report a stable lasing mode accounted for by circulations of a narrow soliton in the cavity, which generates an array of strong pulses (with $10^{3}-10^{4}$ atoms in each, the array's duty cycle being $\simeq 30 \%$ ) when the soliton periodically hits the valve.
\end{abstract}

PACS numbers: 03.75.-b; 03.75.Lm; 05.45.Yv

\section{INTRODUCTION AND THE MODEL}

One of potential applications of Bose-Einstein condensates (BECs) is using them as a source of matter-wave (MW) laser beams. Atomic lasing was predicted and demonstrated in a number of theoretical and experimental works, starting from early ones [1, 2] and recently reviewed in [3]. Especially interesting is a possibility to design a laser operating in a soliton regime, i.e., generating a sequence of narrow spatial packets of coherent atom waves, as proposed, in various settings, in Refs. [4, [5].

In a recent paper [5], we put forward a model of the soliton MW laser based on a set of two tunnel-coupled parallel quasi-one-dimensional (cigar-shaped) traps, one of which is a BEC-filled reservoir, and the other plays the role of a lasing cavity (the setup is shown in Fig. 1 of Ref. [5]), with weakly repulsive and attractive interactions between atoms in the reservoir and cavity, respectively (the sign and size of the respective scattering lengths can be controlled by external magnetic field through the Feshbach-resonance effect [7]; note that static soliton states in dual-core traps, with opposite signs of the scattering lengths in them, were studied in Ref. [8]). In the model, both edges of the reservoir, and the left edge of the cavity are impenetrable, while an outlet (valve) at the right edge of the cavity releases pulses into an outcoupling MW guide. The model was originally designed with an intention to provide for formation of a narrow quasi-soliton that would circulate in the cavity, bouncing from the edges and releasing an outgoing pulse each time that it hits the valve. The circulating soliton is supposed to replenish itself by absorbing atoms pumped from the reservoir.

The model is based on a system of linearly coupled Gross-Pitaevskii equations for the wave functions $\psi(x, t)$ and $\phi(x, t)$ of atoms in the cavity and reservoir, with opposite signs in front of the nonlinear terms. The equations are cast in a normalized form by scaling $\hbar$, atomic mass $m$, and the nonlinearity coefficient in the cavity (in physical units, it is $4 \pi \hbar^{2} a / m$, with $a$ the negative scattering length of atomic collisions) to be 1 , while its counterpart, $\varepsilon$, in the reservoir is small and positive:

$$
\begin{aligned}
i \psi_{t} & =-(1 / 2) \psi_{x x}-|\psi|^{2} \psi-\kappa \phi, \\
i \phi_{t} & =-(1 / 2) \phi_{x x}+\varepsilon|\phi|^{2} \phi-\kappa \psi,
\end{aligned}
$$

with $0<x<L$, i.e., $L$ is the common length of the cavity and reservoir. Keeping $\varepsilon>0$ and the tunnel-coupling coefficient $\kappa>0$ as free parameters, we use the residual scaling invariance of Eqs. (11) and 2 to fix $L \equiv 1$. The model introduced in Ref. [5] also included an external potential and $x$-dependence of the nonlinearity coefficient in equation (11) for the cavity; however, the present analysis demonstrates that stable soliton-lasing regimes can be achieved without these ingredients.

As said above, the model assumes that both edges of the reservoir, and the left edge of the cavity are impenetrable, the respective boundary conditions (b.c.) being $\phi(x=0)=\phi(x=1)=\psi(x=0)=0$. The remaining b.c., corresponding to the valve at the cavity's right edge $(x=1)$ is

$$
\psi_{x}(x=1)=i q \psi(x=1)
$$

with $q>0$ (as explained in Ref. [5], it corresponds to the potential drop $\Delta U=q^{2} / 2$ at the outcoupling edge). After straightforward manipulations with Eqs. (11) and (2), this b.c. yields a balance equation for the total norm of the 
wave functions $\psi$ and $\phi$, which is proportional to the net number of atoms in the traps,

$$
N=\int_{0}^{1}\left(|\psi(x)|^{2}+|\phi(x)|^{2}\right) d x \equiv N_{1}+N_{2} .
$$

The balance equation can be cast in the following compact form:

$$
R(t) \equiv-\frac{d N}{d t}=q|\psi(x=1, t)|^{2} .
$$

Note that the valve gets shut in both limits of $q=0$ and $q \rightarrow \infty$ [in the latter case, Eq. (3) shows that $|\psi(x=1)|^{2} \sim$ $q^{-2}$, hence $R$ vanishes as $\left.1 / q\right]$. Monitoring $R(t)$ by means of Eq. (5), one can characterize the lasing regime (see below). The model does not include an explicit form of the outcoupling waveguide (at $x>1)$, which shapes the released pulses into solitons, as soliton formation in a uniform waveguide is a well-studied problem.

The system is controlled by five parameters: $\varepsilon, \kappa, q$, and initial numbers of atoms, $N_{1}^{(0)}$ and $N_{2}^{(0)}$, in the cavity and reservoir, respectively. It is, of course, difficult to exhaustively explore the corresponding parameter space, looking for physically meaningful regimes. In particular, a lasing mode based on circulation of a well-defined soliton in the cavity was found in Ref. [5] in some parameter region, but it was unstable: after no more than 40 circulations (usually fewer), the soliton would quickly come to a halt, getting much broader. Instead of the unstable circulation mode, a stable regime of periodic release of pulses was found in a large part of the section of the parameter space explored in Ref. 5]. In that regime, a broad "lump" stays put in the cavity, performing cycles of stretching and compression. When its right wing periodically reaches the outlet (valve), weak pulses are released.

The mode of periodic vibrations of the immobile lump is not a spectacular one, and, in practical terms, its drawback is that each pulse generated this way contains a small number of atoms, typically not much larger than 10 [5]. It would be more interesting to find a mode of stable soliton circulations in the cavity. In this paper, we demonstrate in direct simulations that exploration of a broader parameter region reveals a stable soliton-circulation mode. The existence and stability of this mode are directly explained by introducing a simplified analytically tractable version of the model, in which we apply the perturbation theory to the soliton circulating in the cavity. The number of atoms in each released pulse is $N_{\text {pulse }} \sim 10^{3}-10^{4}$, which is quite sufficient to shape the pulses into true MW solitons $\left(N_{\text {pulse }}\right.$ is larger, roughly, by a factor of 100 than in was provided by the above-mentioned pulsation mode in Ref. [5]). The quality of the soliton array is characterized by its duty cycle (the ratio of the temporal width of each pulse to the duration of the cycle), $\simeq 30 \%$, which makes it possible to avoid the unwanted effect known (in terms of telecommunications [6]) as inter-symbol interference (conspicuous overlap between pulses through their extended tails); in the regime investigated in Ref. [5], the duty cycle was $\simeq 50 \%$.

The rest of the paper is organized as follows. In Section II, we display generic examples of two different stable intracavity-circulation regimes, periodic and quasi-periodic ones, found in direct simulations of Eqs. (1), (1), which provide for the MW lasing in the soliton format (we also display an example of another generic regime possible in the system, viz., persistent irregular circulations). In Section III, we summarize results of the systematic analysis in the form of stability charts for the periodic and quasi-periodic circulation modes in a relevant parameter space, and present dependences of essential characteristics of the modes on parameters of the system. The simplified version of the model and its analytical perturbative solutions, that demonstrates the existence of the stable-circulation regime, are presented in Section IV. The paper is concluded by Section V, where, in particular, we give estimates for physical characteristics of the proposed pulsed MW laser.

\section{STABLE CIRCULATION MODES}

Numerical simulations of Eqs. (1) and (2) were performed by means of a finite-element pseudospectral method. In most cases, initial configurations were built (following Ref. [5]) as stationary solutions to the same equations, but with b.c. (3) replaced by $\psi(x=1)=0$.

Exploring the parameter space of the model in a more systematic way than in Ref. [5], we were able to find three distinct types of regimes featuring persistent circulations of a narrow soliton in the cavity and, accordingly, the MW lasing in the pulsed format. The first type is persistent in the sense that the soliton keeps to circulate as long as the depletion of the reservoir does not become conspicuous, but the circulations are irregular (quasi-random). An example of this regime is shown in Fig. 1 in terms of the evolution of the density profile in the cavity, $|\psi(x, t)|^{2}$, and the corresponding release rate, $R(t)$. We do not report more details about this mode, as it seems unusable for applications.

More promising are two other varieties of the circulation mode, one periodic and another one quasi-periodic. Typical examples of spatiotemporal patterns specific to these modes are displayed in Figs. 2] and 3 by means of both 


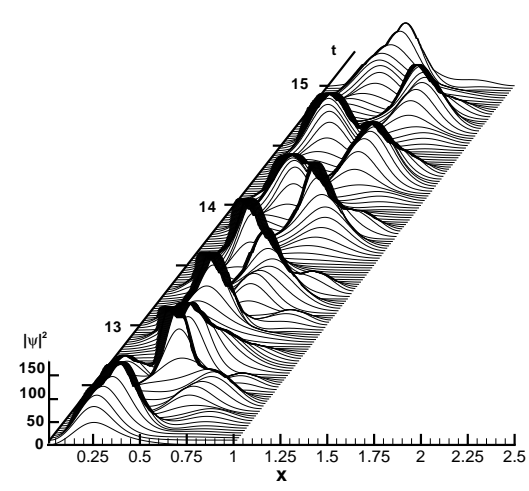

(a)

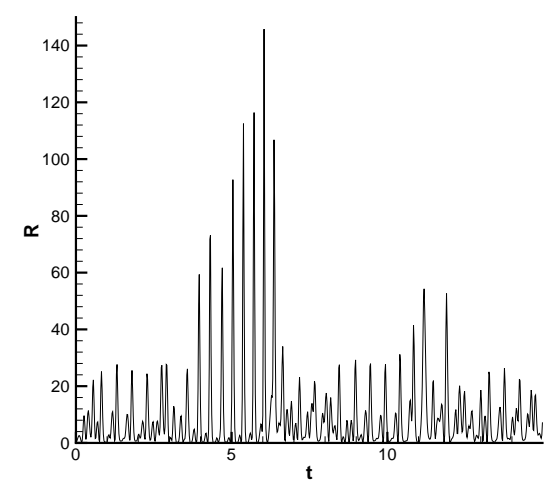

(b)

FIG. 1: A typical example of the spatiotemporal density distribution in the cavity, $|\psi(x, t)|^{2}$ (a), and the corresponding release rate, defined by Eq. (5), as a function of time (b), in the case of persistent irregular circulations of the intra-cavity soliton circulations. Parameters are $\kappa=0.8, \varepsilon=0.0025, q=32.5$, and initial values of the norm of the wave function in the cavity and reservoir are $N_{1}^{(0)}=0$ and $N_{2}^{(0)}=1000$.

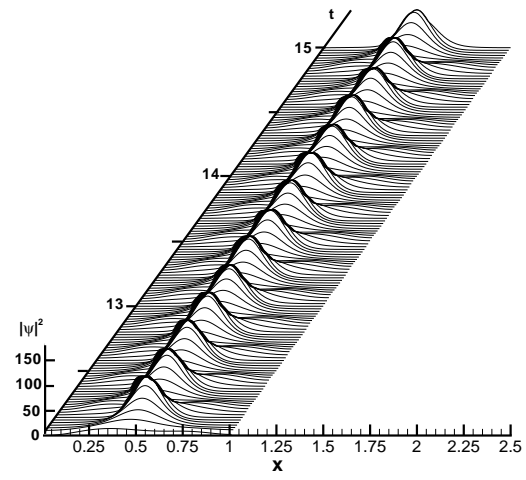

(a)

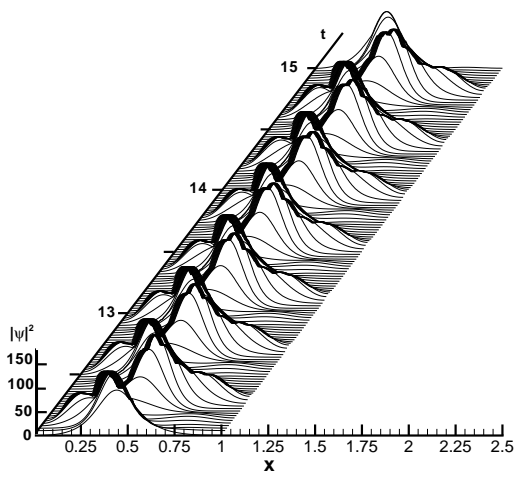

(b)

FIG. 2: Generic examples of the spatiotemporal density distribution in the cavity, $|\psi(x, t)|^{2}$, in regimes of periodic (a) and quasiperiodic (b) intra-cavity soliton circulations, for $N_{1}^{(0)}=10, N_{2}^{(0)}=1000, \kappa=0.8, \varepsilon=0.002$, and (a) $q=12.5$, (b) $q=21.0$.

spatiotemporal profiles of $|\psi(x, t)|^{2}$ and respective contour plots. The corresponding temporal patterns of $R(t)$, which determine the profile of the soliton streams released from the cavity (the same way as the temporal shape of an input optical signal coupled into a nonlinear fiber determines the form of the soliton array created in it [6]), are displayed in Fig. 4 It is seen that both modes, periodic and quasi-periodic ones, readily establish themselves after a transient (this self-establishment was adopted as a criterion of the mode's stability). In the former case, the soliton circulates in the central part of the cavity, while in the quasi-periodic mode it sweeps the entire domain and strongly hits the valve, in an almost periodic fashion; for this reason, the amplitude of the generated pulses in essentially higher in the latter case. Another notable difference of the quasi-periodic regime from its periodic counterpart is period doubling obvious in Fig. 3(b), due to which each cycle of the quasi-periodic oscillations is composed of a narrow tall pulse to which a small hump is attached.

An additional relevant characteristic of the periodic and quasi-periodic regimes is given in terms of the time 


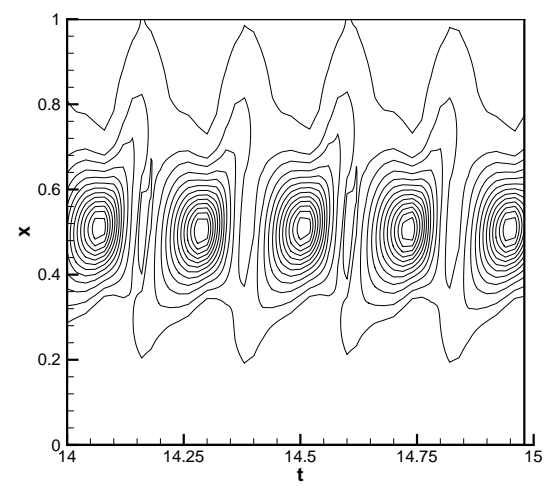

(a)

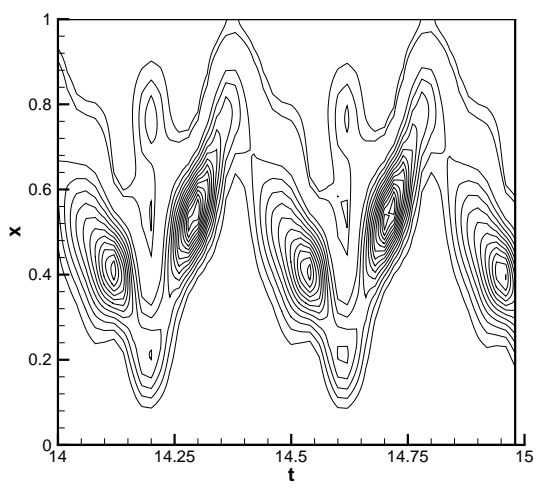

(b)

FIG. 3: The same as in Fig. 2 shown by means of contour plots for the spatiotemporal field of $|\psi(x, t)|^{2}$.

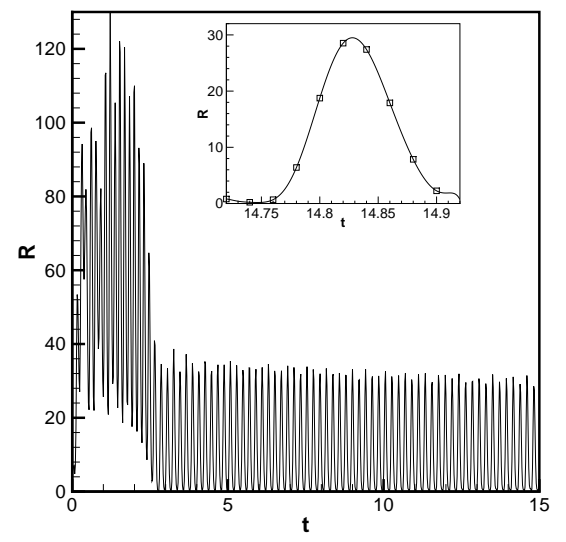

(a)

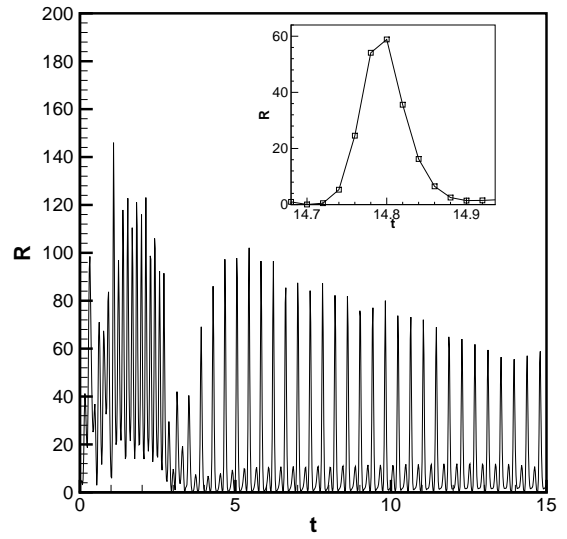

(b)

FIG. 4: The release rate $R$, defined as per Eq. (5], vs. time, in the same cases as in Figs. 2 and 3 Insets in each panel show the shape of individual pulses in the established regime, with the continuous lines being a guide to the eye. The duty cycle of the (quasi-) periodic pulse arrays, defined as the ratio of the full time width of the temporal pulse at its half-maximum to the full period, is $32 \%$ (a) and $27 \%$ (b).

dependence of norms $N_{1}$ and $N_{2}$ in the cavity and reservoir, as well as the total norm, $N_{1}+N_{2}$ [see Eq. (41)]. For the same cases as shown in Figs. 2] and 3] the dependences are displayed in Fig. [5] They clearly highlight the character of the dynamical regimes: a relatively fast matter exchange between the reservoir and cavity, and slower depletion of the total norm, which actually happens by jumps when a new pulse is released into the outcoupling MW waveguide.

All stable periodic and quasi-periodic soliton-lasing modes found at other values of parameters (see stability charts presented in the next section) are very similar to the examples displayed in Figs. 2 - 5] As concerns the persistent irregular regimes, they are quite similar to the example displayed in Fig. 11). 


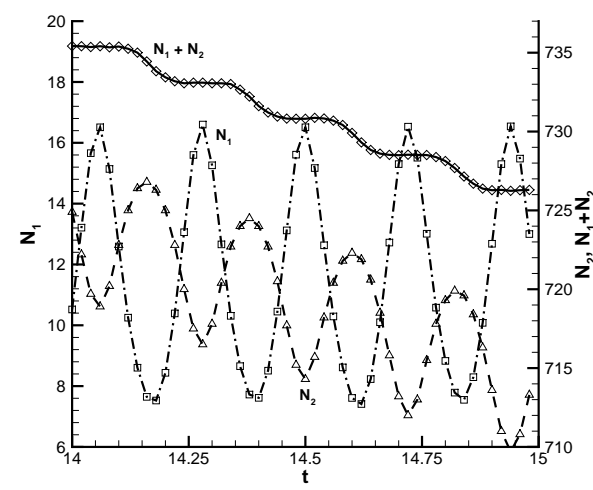

(a)

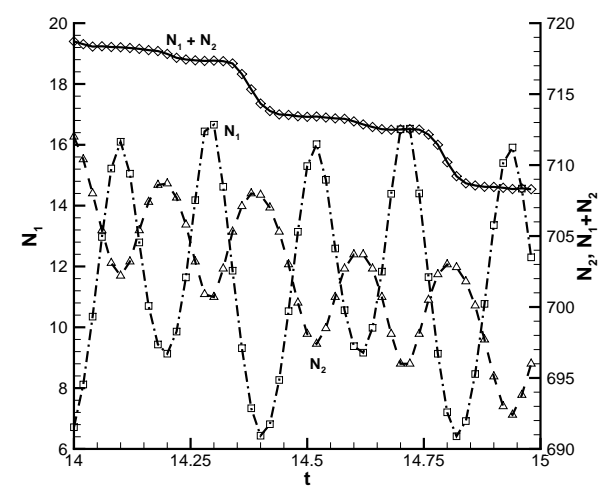

(b)

FIG. 5: The evolution of the total norm, $N_{1}+N_{2}$ (rhombuses), and norms in the lasing cavity and reservoir, $N_{1}$ (squares) and $N_{2}$ (triangles), in the same established dynamical regimes [periodic and quasiperiodic ones, (a) and (b)] which are shown in Figs. 2 and 3

\section{STABILITY MAPS FOR THE CIRCULATION MODES.}

As the model contains several parameters, it is necessary to identify those which strongly affect the stability of the lasing modes. For this purpose, we summarize results of a large number of simulations in charts displayed in Figs. [6] and 7 The periodic and quasi-periodic circulation modes are stable, respectively, inside the area bounded by the continuous line, and between dashed and continuous borders. The charts displayed in these figures were obtained by varying $q$ and $\varepsilon$ in steps of 2 and 0.001 , respectively, unless strong sensitivity to variation of the parameters dictated to use reduced steps.

Comparison of Figs. 6(a) and 6(b), which differ by values of the initial number of atoms in the cavity, demonstrates that the outer stability border (appertaining to the quasi-periodic regime) is relatively insensitive to this parameter, while the inner border, which separates the stability areas of the periodic and quasi-periodic regimes, is conspicuously affected by the initial conditions. The effect of variation of the strength of the linear coupling between the reservoir and cavity, $\kappa$, on the stability region is illustrated by comparison of Figs. 6 b) and 7 Naturally, the decrease of $\kappa$ entails shrinkage of the stability area, as it becomes harder for the circulating soliton to replenish itself by collecting matter pumped from the reservoir (see also the analytical model presented in Section V). In addition, the results suggest that, at smaller $\kappa$, the stability region shifts to higher values of $q$. Detailed analysis shows that the increase of $q$ makes the release rate $R$ smaller in this case, thus compensating the decrease of the pumping rate due to the reduction in the coupling strength.

A noteworthy peculiarity of Fig. [6] is a nearly flat lower stability border, which is found at $q=q_{\min } \approx 4$. An explanation for this flat border is provided by the analytical model presented below.

Beneath lower borders of the stability areas in Figs. 4 and 5, no circulation regime is possible. Above the upper stability borders, persistent irregular circulations take place, similar to the example shown in Fig. 2 Outside the stability area, there is an additional border between the no-circulation and irregular-circulation regimes, which we do not display here, as it is of little relevance to applications.

Effects of the variation of control parameters on characteristics of the generated pulse arrays were investigated too. In particular, a change in $N_{2}^{(0)}$ does not affect the results in any tangible way, and arrays of the outcoupling pulses are quite insensitive to the variation of $\varepsilon$ too. On the other hand, the dependence on the valve parameter $q$ is conspicuous, as seen in Fig. 8

\section{THE ANALYTICAL MODEL}

A qualitative explanation to the stable regime of periodic circulation of the soliton in the cavity may be provided by the perturbation theory, which, at the zero order, assumes shuttle oscillations of a soliton in the cavity of length 


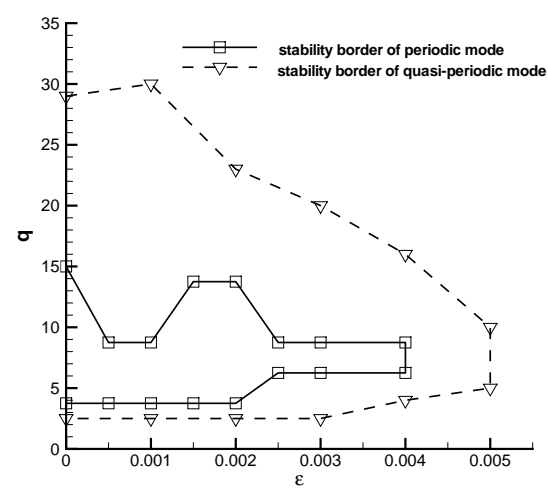

(a)

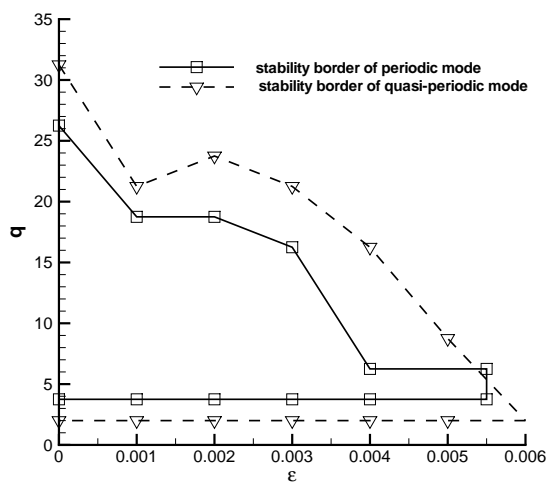

(b)

FIG. 6: Stability borders of the periodic and quasi-periodic regimes in the parameter plane $(\varepsilon, q)$, for $\kappa=0.8, N_{2}^{(0)}=1000$, and (a) $N_{1}^{(0)}=0$, (b) $N_{1}^{(0)}=10$.

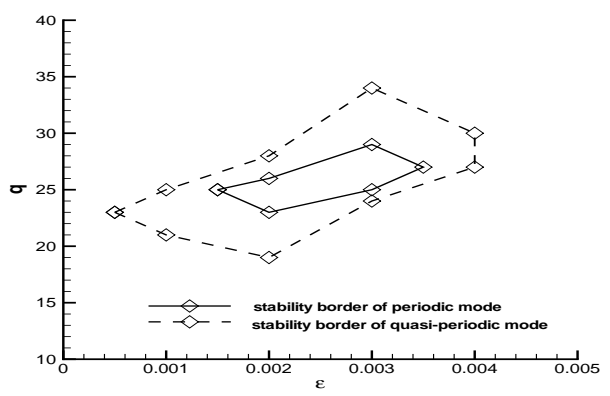

FIG. 7: The same as in Fig. 4(b), except that $\kappa=0.5$.

$L$ with zero b.c., $\psi(x=0)=\psi(x=L)=0$, with no coupling to the reservoir, $\kappa=0$. The respective zero-order approximation for the soliton is

$$
\begin{aligned}
\psi_{0}(x, t) & =\eta \operatorname{sech}\left(\eta(x-\xi(t)) e^{i v x+i \Phi(x)},\right. \\
\frac{d \xi}{d t} & =v, \frac{d \Phi}{d t}=-\frac{1}{2}\left(v^{2}-\eta^{2}\right),
\end{aligned}
$$

where $\eta$ and $v$ are its amplitude and instantaneous velocity. In this approximation, Eq. (11) conserves two dynamical invariants, viz., the norm, $N_{1}=\int_{0}^{L}|\psi(x)|^{2} d x$, see Eq. (4) (in this section, we do not set $L=1$, to present the dependence of the results on parameters in a clearer form), and the energy (Hamiltonian),

$$
H_{1}=\frac{1}{2} \int_{0}^{L}\left(\left|\psi_{x}\right|^{2}-|\psi|^{4}\right) d x .
$$

Note that, for unperturbed soliton (6), the values of these invariants are

$$
\left(N_{1}\right)_{\mathrm{sol}}=2 \eta, \quad\left(H_{1}\right)_{\mathrm{sol}}=\eta v^{2}-\frac{1}{3} \eta^{3} .
$$

Zero b.c. imply the presence of formal mirror images of the soliton beyond the points of $x=0$ and $x=L$, i.e., $\psi(-x)=-\psi(x)$, and $\psi(x)=-\psi(2 L-x)$, respectively. As is well known, a pair of solitons with opposite signs repel each other [9], which explains bounces of the soliton from edges of the cavity and its shuttle motion. 


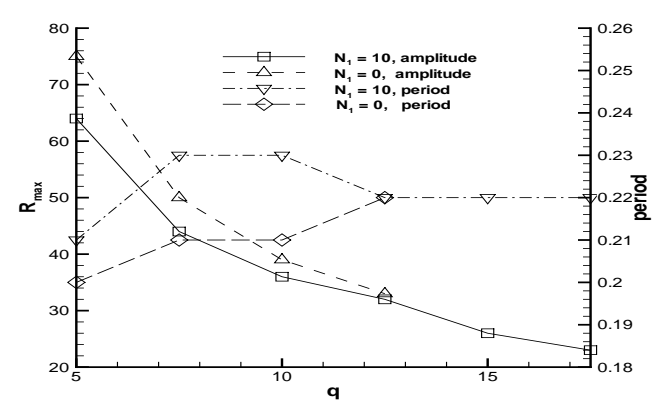

FIG. 8: The maximum value ("amplitude") of the release rate $R$, and its oscillation period, versus the outcoupling coefficient $q$, for $\kappa=0.8, \varepsilon=0.002, N_{2}^{(0)}=1000$, and two different values of $N_{1}^{(0)}$. Data sets for $N_{1}^{(0)}=0$ terminate at points beyond which stable circulation modes are not found.

In the first nontrivial approximation, we treat the coupling coefficient $\kappa$ and b.c. coefficient $q$ in Eq. (3) as small parameters. To predict the possibility of stable periodic circulations of the soliton in this case, we will treat it as a quasiparticle trapped in an infinitely deep potential box, between $x=0$ and $x=L$. Then, the shuttle motion can be predicted by means of balance equations for the norm and energy [this approach was elaborated in detail for a kink (topological solitons) moving as a shuttle in models based on a perturbed sine-Gordon equation [9]].

First, note that Eq. (5) makes it possible to calculate the loss of norm $N_{1}$ for the soliton bouncing from the right edge $(x=L)$ :

$$
\left(\Delta N_{1}\right)_{q}=-q \int_{-\infty}^{+\infty}|\psi(x=L, t)|^{2} d t=-2 \frac{q \eta}{v}
$$

where $\psi(x=L)$ was taken as per the unperturbed soliton (6). As concerns the Hamiltonian, in the absence of perturbations its conservation follows from the continuity equation relating the Hamiltonian density and current conjugate to it,

$$
\frac{\partial}{\partial t}\left[\frac{1}{2}\left(\left|\psi_{x}\right|^{2}-|\psi|^{4}\right)\right]=\frac{i}{4} \frac{\partial}{\partial x}\left[\left(\psi_{x}^{*} \psi_{x x}-\psi_{x} \psi_{x x}^{*}\right)+2|\psi|^{2}\left(\psi \psi_{x}^{*}-\psi^{*} \psi_{x}\right)\right]
$$

Integration of this equation over the domain of $0<x<L$ gives rise to the evolution equation for $H_{1}$ similar to its counterpart (5) for the norm,

$$
\frac{d H_{1}}{d t}=q\left[|\psi|^{4}+\frac{1}{4}\left(\psi_{x x} \psi^{*}+\psi_{x x}^{*} \psi\right)\right] .
$$

Substituting unperturbed soliton (6) in this relation and subsequent integration yield the net change of $H_{1}$ induced by the bounce of the soliton from the valve, cf. Eq. (9):

$$
\left(\Delta H_{1}\right)_{q} \equiv q \int_{-\infty}^{+\infty} \frac{d H_{1}}{d t} d t=\frac{q \eta}{v}\left(\eta^{2}-v^{2}\right) .
$$

The change in the norm and energy incurred by the collision of the soliton with the valve is to be compensated by matter which was pumped from the reservoir and absorbed by the soliton sweeping the cavity. Assuming, in the spirit of the perturbation theory, that the matter-wave background built up in the cavity between passages of the soliton, $\psi_{0}(x)$, has a small amplitude, and the soliton collects all the available matter, the linearization of Eq. (1) yields

$$
\psi_{0}(x)=i \kappa \phi_{0}(x) \tau(x)
$$

where $\tau(x)$ is the time between two passages of the soliton through a given point, and

$$
\phi_{0}(x)=\sqrt{\frac{2 N_{2}}{L}} \sin \left(\frac{\pi x}{L}\right)
$$


is the ground-state wave function in the reservoir, with the number of atoms $N_{2}$ in it; the depletion of the reservoir is neglected, which is a natural assumption in the perturbative setting. As the soliton passes each point twice in the course of the cycle of circulations, it is easy to see that $\tau(x)$ has two branches,

$$
\tau_{1}=2 x / v, \tau_{2}=2(L-x) / v .
$$

Then, it follows from Eqs. (4), (7) and (14) that the norm and energy collected by the soliton within the circulation cycle are

$$
\begin{aligned}
& \left(\Delta N_{1}\right)_{\kappa}=\left(\kappa \phi_{0}\right)^{2} \int_{0}^{L}\left[\tau_{1}^{2}(x)+\tau_{2}^{2}(x)\right] d x=C_{N} \frac{\kappa^{2} N_{2} L^{2}}{v^{2}} \\
& \left(\Delta H_{1}\right)_{\kappa}=-\frac{1}{2}\left(\kappa \phi_{0}\right)^{4} \int_{0}^{L}\left[\tau_{1}^{4}(x)+\tau_{2}^{4}(x)\right] d x=-C_{H} \frac{\left(\kappa^{2} N_{2}\right)^{2} L^{3}}{v^{4}}
\end{aligned}
$$

where $C_{N} \equiv 4\left(\frac{1}{3}-\frac{1}{\pi^{2}}\right) \approx 0.93$ and $C_{H}=\frac{24}{5}-\frac{30}{\pi^{2}}+\frac{189}{4 \pi^{4}} \approx 2.25$.

The periodic circulations are provided by obvious balance conditions, $\left(\Delta N_{1}\right)_{q}+\left(\Delta N_{1}\right)_{\kappa}=0$ and $\left(\Delta H_{1}\right)_{q}+\left(\Delta H_{1}\right)_{\kappa}=$ 0. Using Eqs. (9), (11) and (15), (16), these conditions determine equilibrium values of the soliton's amplitude and velocity, $\eta_{0}$ and $v_{0}$ :

$$
\begin{aligned}
& \eta_{0}^{2}=\frac{C_{N}}{2} \frac{\kappa^{2} N_{2} L^{2}}{q} \\
& v_{0}=\eta_{0}-\delta v_{0}, \delta v_{0} \equiv \frac{C_{H}}{2} \frac{\left(\kappa^{2} N_{2}\right)^{2} L^{3}}{q \eta^{5}}
\end{aligned}
$$

Within the framework of the analytical model it is also possible to analyze stability of this equilibrium solution, by assuming small deviations of $\eta$ and $v$ from values (17) and (18). For the corresponding nonequilibrium solutions, Eqs. (9), (11) and (15), (16) give rise to a map, $2 \eta \rightarrow 2 \eta^{\prime}=2 \eta+\left(\Delta N_{1}\right)_{q}+\left(\Delta N_{1}\right)_{\kappa}, H_{1} \rightarrow H_{1}^{\prime}=H_{1}+\left(\Delta H_{1}\right)_{q}+\left(\Delta H_{1}\right)_{\kappa}$ [in fact, it is more convenient to analyze the map written in terms of $\eta$ and reduced Hamiltonian, $\left.h \equiv H_{1}-(2 / 3) \eta^{3}\right]$. Then, equilibrium solution (17) and (18) is a fixed point (FP) of the map, and its stability is determined by multiplicators $\mu$, i.e., eigenvalues of the map linearized around the FP. Direct calculation yields two eigenvalues, $\mu_{\eta}=1-2\left(q / \eta_{0}\right)$ and $\mu_{h}=1-\left(q / \eta_{0}\right)$. As $q$ is a small positive parameter, we conclude that $0<\mu_{\eta}, \mu_{h}<1$, hence the FP is stable indeed.

Despite many approximations adopted in the analytical version of the model, the prediction for the soliton's amplitude given by Eq. (17) generally agrees with numerical results. For instance, in the example of the periodic regime displayed in Figs. 2(a), 31(a) and 4 (a), the averaged maximum density of the solitonic waveform is $\left\langle\left(\left|\psi\left(x,\left.t\right|^{2}\right){ }_{\max }\right\rangle \simeq\right.\right.$ 27, while Eq. (17) predicts, for the same case, $\eta_{0}^{2} \approx 25$.

The analytical model also helps to explain the presence of the lower border of the stability region in Fig. 6] Indeed, stable circulations of the soliton are possible if the release of matter through the outlet can compensate the permanent inflow of matter from the reservoir. Adopting a rough estimate according to which $\eta, v$ and $q$ are on the same order of magnitude, which is borne out by numerical data in the general case, and making use of Eqs. (9) and (15), one can put the latter condition in the form of $q^{3}>q_{\min }^{3} \sim \kappa^{2} N_{2} L^{2}$. For parameter values corresponding to Fig. 6] this yields $q_{\min } \sim 7$, which generally agrees with the situation shown in that figure.

One can also understand why the lower stability border in Fig. 6] is nearly flat (in fact, the explanation is quite a general one, and is not predicated on the validity of the above analytical model). Indeed, it follows from Eq. (2) that nonlinear deformation of ground state (13) is negligible under condition $\varepsilon \ll \varepsilon_{\max } \equiv \pi^{2} /\left(N_{2} L\right)$. In the case corresponding to Fig. 6] with $N_{2}=1000$ and $L=1$, one obtains $\varepsilon_{\max } \simeq 0.01$, which complies with what one can observe in Fig. 6]

A limitation of the quantitative validity of results produced by the analytical model can be seen from the calculation of the maximum value of the matter-release rate: Eqs. (5), (6) and (17) yield a perturbation-theory result for it, $R_{\max }=\left(C_{N} / 2\right) \kappa^{2} N_{2} L^{2}$, which does not depend on the outlet parameter, $q$. On the other hand, Fig. 8 shows that, in the range of $5<q<17.5$, the numerical results yield an approximate dependence, $R_{\max } \sim q^{-0.75}$. However, the perturbation theory does not really apply in this range.

\section{CONCLUSION}

In this paper, we have investigated the model of the matter-wave laser generating a periodic array of coherent pulses, which was recently introduced in Ref. [5]. While only a relatively primitive regime of vibrations of a broad immobile 
lump in the cavity was found in the original consideration of the model, here we have demonstrated that exploration of a broader parameter region reveals stable lasing modes accounted for by periodic or quasi-periodic circulations of a narrow stable soliton in the cavity; the soliton periodically generates an outcoupling pulse, hitting the valve, and then gets replenished, absorbing matter pumped from the reservoir. The existence of the stable circulation regime, and some features of the numerically found stability regions were explained in the framework of a simplified analytical model, that treated the intra-cavity soliton as a quasi-particle, and predicted the stable regime by means of balance conditions for the number of atoms and energy in the cavity.

Getting back from the Gross-Pitaevskii equations in the normalized form of Eqs. (11), (2) to physical units, and making use of typical values of physical parameters for the ${ }^{7} \mathrm{Li}$ condensate, it is easy to conclude that the circulation regime found in this work easily provides for the generation of regular pulses built of $\mathcal{N}=10^{3}-10^{4}$ atoms each (the vibrational lasing mode investigated in Ref. [5] gave rise to $\mathcal{N}$ smaller by a factor of $\sim 100$ ). With the scaled value of the initial norm, $N_{2}^{(0)}=1000$, employed in the examples, the laser would generate $\sim 100$ pulses before the reservoir depletion will becomes tangible. However, the latter number may be readily made indefinitely large, as indefinite increase of $N_{2}^{(0)}$ does not affect the stability of the circulation regime.

Undoing the rescaling which cast the Gross-Pitaevskii equation in the normalized form of Eq. (1), it is easy to see that, for an experimentally relevant value of the cavity length, $L=100 \mu \mathrm{m}$, the time unit in the normalized equation corresponds to physical time $t_{0}=m L^{2} / \hbar \approx 1 \mathrm{~s}$ (recall $m$ is the atomic mass, i.e., $m \approx 1.2 \times 10^{-26} \mathrm{~kg}$ for ${ }^{7} \mathrm{Li}$ ). Therefore, according to Fig. [8] a typical circulation period is $\simeq 0.2 \mathrm{~s}$ in physical units. Lastly, detailed consideration of numerical data shows that the typical value of the duty cycle characterizing the quality of the generated pulse array is $\simeq 30 \%$ (the quality of small-amplitude arrays found in Ref. [5] was poorer, with the duty cycle $\sim 50 \%$, i.e., the pulses were more overlapped than in the circulation regime).

The realization of the proposed matter-wave soliton laser by means of available experimental techniques seems to be quite feasible. It may also be possible to consider another variant of the proposed matter-wave soliton laser, with outlets at both edges at the cavity. The so modified system may generate a double stream of matter-wave solitons, which, however, needs separate consideration.

\section{Acknowledgement}

The work of B.A.M. was supported, in a part, by the Israel Science Foundation through the Center-of-Excellence grant No. 8006/03. 
[1] Holland M, Burnett K, Gardiner C, Cirac J I, and Zoller P 1996 Phys. Rev. A 54 R1757; Moy G M and Savage C M 1997 ibid. 56 R1087; Kneer B, Wong T, Vogel K, Schleich W P, and Walls D F 1998 ibid 58 4841; Band Y B, Julienne P S and Trippenbach M 1999 ibid. 59 3823; Martin J L, McKenzie C R, Thomas N R, Sharpe J C, Warrington D M, Manson P J, Sandle W J and Wilson A C 1999 J. Phys. B - At. Mol. Opt. Phys. 323065

[2] Mewes M O, Andrews M R, Kurn D M, Durfee D S, Townsend C G, and Ketterle W 1997 Phys. Rev. Lett. 78 582 Miesner H J, Stamper-Kurn D M, Andrews M R, Durfee D S, Inouye S and Ketterle W 1998 Science 279 1005; Bloch I, Hansch T W and Esslinger T 1999 Phys. Rev. Lett. 82 3008; Deng L, Hagley E W, Wen J, Trippenbach M, Band Y, Julienne P S, Simsarian J E, Helmerson K, Rolston S L and Phillips W D 1999 Nature 398 218; Hagley E W, Deng L, Kozuma M, Wen J, Helmerson K, Rolston S L and Phillips W D 1999 Science 2831706

[3] Bongs K and Sengstock K 2004 Rep. Progr. Phys. 67907

[4] Leboeuf P, Pavloff N, and Sinha S 2003 Phys. Rev. A 68 063608; Carr L D and Brand J 2004 Phys. Rev. A 70 033607; Rodas-Verde M I, Michinel H and Pérez-García V M 2005 Phys. Rev. Lett. 95153903

[5] Chen P Y P and Malomed B A 2005 J. Phys. B - At. Mol. Opt. Phys. 384221

[6] Agrawal G P, Fiber-Optic Communication Systems (John Wiley \& Sons, Inc.: New York, 1997)

[7] Kagan Y, Surkov E L, and Shlyapnikov G V 1997 Phys. Rev. Lett. 79 2604; Roberts J L, Claussen N R, Burke J P, Jr, Greene C H, Cornell E A, and Wieman C E 1998 ibid. 81 5109; Inouye S, Andrews M R, Stenger J, Miesner H-J, Stamper-Kurn D M, and Ketterle W 1998 Nature 392151

[8] Shchesnovich V S, Malomed B A, and Kraenkel R A 2004 Physica D 188 213; Shchesnovich V S, Cavalcanti S B, and Kraenkel R A 2004 Phys. Rev. A 69033609

[9] Kivshar Y S and Malomed B A 1989 Rev. Mod. Phys. 61763 